\title{
Erratum
}

\section{Concise Formal Synthesis of (+)-Englerin A and Total Synthesis of (-)-Orientalol F: Establishment of the Stereochemistry of the Organocatalytic [4+3]-Cycloaddition Reaction}

Chao-Lei Wang, Bing-Feng Sun,* Shu-Guang Chen, Rui Ding, Guo-Qiang Lin,* Jin-Yi Xu,* Yong-Jia Shang Synlett 2012, 23, 263.

The authors noticed that the absolute configuration of catalyst 7 employed in the [4+3]-cycloaddition reaction was incorrect. $(S, S)-7$ should be $(R, R)-7$ throughout the article. Thus, the following parts should be corrected:

1. Page 263, right column, line 13 from bottom.

2. Page 264, structure in Scheme 1.

3. Page 265, left column, line 6 from bottom.

4. Page 265, structure in Scheme 5.

The correct structure of $(R, R)-7$ is shown below.

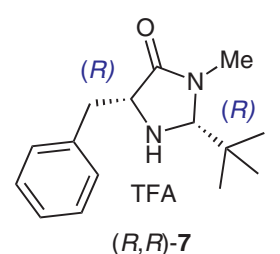

The authors apologize for this mistake and for any inconvenience it may have caused. 